\title{
Psychotherapy across Languages: beliefs, attitudes and practices of monolingual and multilingual therapists with their multilingual patients
}

\author{
Beverley Costa $^{1}$ Mothertongue \\ Jean-Marc Dewaele University of London
}

\begin{abstract}
The present study investigates beliefs, attitudes and practices of 101 monolingual and multilingual therapists in their interactions with multilingual patients. We adopted a mixed-method approach, using an on-line questionnaire with 27 closed questions which were analysed quantitatively and informed questions in interviews with one monolingual and two multilingual therapists. A principal component analysis yielded a four-factor solution accounting for $41 \%$ of the variance. The first dimension, which explained $17 \%$ of variance, reflects therapists' attunement towards their bilingual patients (i.e. attunement versus collusion). Further analysis showed that the 18 monolingual therapists differed significantly from their 83 bi- or multilingual peers on this dimension. The follow up interviews confirmed this result. Recommendations based on these findings are made for psychotherapy training and supervision to attend to a range of issues including: the psychological and therapeutic functions of multi/bilingualism; practice in making formulations in different languages; the creative therapeutic potential of the language gap.
\end{abstract}

\section{Introduction}

Migration, acculturation processes, living with plural worldviews and identities and communicating across languages are all experiences which permeate contemporary communities. Increasingly, people are moving across borders in pursuit of work, safety and refuge. An inevitable consequence of this is that there are many people accessing services, including counselling and psychotherapy services, who do not speak the official language of the country in which they find themselves. In London alone it is estimated that over 300 languages are spoken by schoolchildren (Burck, 2004, p. 315). To complement this, the number of multilingual people training to be therapists has increased in recent years. The current data does not present a very clear picture but where organizations keep data on therapists' multilingualism, the current situation (2012) for active members registered with the United Kingdom Council for Psychotherapy, for

${ }^{1}$ Correspondence concerning this article should be addressed to Beverley Costa. E-mail: Beverley@mothertongue.org.uk 
example, shows that 1,298 are able to conduct therapy in more than one language out of a total active membership of 7,085. For the purposes of this paper, we use Li Wei's (2000) definition of the term bilingual, namely "describ[ing] someone with the possession of two languages" (p. 7), but he also states that it can cover any number of languages. We do not make a distinction between bi- and multilinguals, so all speakers of more than one language will be included in the category of the multilingual. We use the terms "client" and "patient" to refer to the users of mental health clinical services.

Increasingly therapists are becoming aware of the psychotherapeutic implications of being multilingual both for the patient and for the therapist. It is an area that straddles the disciplines of psychotherapy and linguistics. This research paper attempts to reflect this by adopting a multi-disciplinary approach: the researchers are from the two different disciplines of Applied Linguistics and Psychotherapy. Although there is increasing interest, the role of language in therapy for multilingual patients and for multilingual therapists has attracted relatively little investigation compared with the amount of interest dedicated to the role of culture in therapy. It is, of course, difficult to separate out language from culture but the aim of this research is to focus as closely as possible on language. This is an area which merits attention and consideration not least because many therapists may share Perez Foster's (1998) early concerns that work in English with non native English speakers could be: "a pseudotherapy" which simply sides with the patient's resistance to the mother tongue and the mother era, or a "quasitherapy" where the essential material is lost in the complex cognitive traffic of bilingualism..." (p. 202). Some therapists may not even consider this as a potential issue and will not address their patients' choice of language at all. Sometimes it is the patient who is left to ponder on its meaning. The following is a self-report from a patient interviewed in Dewaele (2010, p. 204). The patient's mother tongue was Greek and her next additional language was English:

I think when I talk about emotional topics I tend to code-switch to English a lot. I remember when I was seeing a psychologist in Greece for a while I kept codeswitching from Greek to English. We never really talked about this...To my mind it may have been some distancing strategy.

Patients may feel distressed as a result of unacknowledged language proficiency differentials between the patient and the therapist. The migrants who took part in the European study "Health for all, all in health" were asked about their experiences with mental health care. They indicated that the healthcare providers underestimated their language issues and that language barriers resulted in greater feelings of paranoia and aggression during their encounters with healthcare providers (De Maesschalck, 2012).

Although, as already stated, there is relatively little written about the experience of multilinguals in psychotherapy, there are some notable exceptions. For example, AmatiMehler, Argentieri and Canestri (1993) consider the issues from a psychoanalytic 
perspective and draw principally from case material with both clients and clinicians who are bi/multilingual (see also Altarriba \& Santiago-Rivera, 1994; Santiago-Rivera, Altarriba, 2002; Schrauf, 2000). More recent research includes Bowker and Richards (2010) and Stevens and Holland (2008) who focus their research principally on monolingual therapists working with bi/multilingual clients. Our research paper focuses on a comparison between monolingual and multilingual therapists in order to identify possible differences between the way they operate across languages, when they share a native language or when they do not share a native language with their patient. Our purpose is to discover what can be learned from each other about working effectively with multilingual patients, which could benefit the practice of psychotherapists working across languages. Amati-Mehler et al. (1993) feel that it is important to include other theoretical models besides psychoanalysis although as previously stated, the researchers aim "...to set out the difficulties facing those who try to deal with the subject of multilingualism from one specific angle - in this case of psychoanalysis. As can be seen... there are many queries to be answered, numerous disciplines are involved, and various theories can be used" (p. 221). We have chosen to conduct our research with therapists from a wide range of theoretical orientations.

\section{Language and Psychotherapy}

The psychoanalytic concept of splitting has a particular relevance for people who are bilingual. Splitting can be defined and understood in many different ways in the different theoretical models of the psyche. For our purposes here, we refer to the process of separating the self from difficult emotions and experiences in order to defend from pain. This can serve a protective function or it can result in a distorted view and disconnection from the self and others. With regard to multilingualism Amati-Mehler (1993, p. 264) view this not as the cause of splitting but that "splitting processes lean on and in a certain way exploit the different linguistic registers as a means for organising and expressing themselves" (p. 264). An excellent example of this is given by the examination by Patrick Casement (1992, as cited in Amati-Mehler, 1993, p. 176) of Samuel Beckett's bilingualism stating that "the only expedient by which he could gain his internal freedom and chances for creativity was, in Casement's opinion, the repudiation not only of his mother and his motherland, but also and above all of his mother tongue". It was by writing in French (Beckett's second language) that Beckett was able to find his creative voice. As the example of Beckett demonstrates, multilingualism in itself need not be the cause of difficulty and hence the split. It may, however, provide a means whereby the splitting can occur and may provide expressive as well as defensive opportunities.

For people who are multilingual, the way in which experiences and emotional reactions are encoded becomes more complex when more than one language is spoken. One of the ways in which multilinguals cope is by splitting and creating new selves for each of the languages spoken. Priska Imberti (2007, p. 71) who migrated from Argentina to New York as a young woman refers to the new self she had to create - "When we change languages, both our worldview and our identities get transformed. We need to become new selves to speak a language that does not come from our core self, a language that does not reflect our inner-connectedness with the culture it represents". Pavlenko (2006) 
investigated the question whether multilinguals feel that they become different people when they change languages. She also looked at how they make sense of these perceptions and what prompts some to see their language selves as different. Her analysis of the feedback of 1039 multilinguals on an open question about "feeling different in a foreign language" revealed that two thirds of participants reported feeling different when using another language. Participants linked their perceptions of different selves to four causes: "(1) linguistic and cultural differences; (2) distinct learning contexts; (3) different levels of language emotionality; (4) different levels of language proficiency" (p. 10). Pavlenko concluded that the perception of different selves is not restricted to immigrant multilinguals, but is part of the general multilingual experience. She also cautions that "similar experiences (e.g. change in verbal and non-verbal behaviours accompanying the change in language) may be interpreted differently by people who draw on different discourses of bi/multilingualism and self" (p. 27).

Wilson (2008) investigated the relationship between the extent to which multilinguals felt different when switching language and their personality profiles. She found that introverts were more likely to feeling different when operating in an L2 when they had at least intermediate or advanced proficiency in the L2. Participants who had learned their L2 at a younger age were more likely to feel differently. Differences in a felt sense of self were also explored by Ozanska-Ponikwia (2011) to include different ways of expression of emotion in differing languages by Polish immigrants in English-speaking countries. She argues that most people feel different when using a second language but that some are more aware of it than others, especially those with higher levels of emotional intelligence.

Dewaele and Nakano (2012) looked at multilinguals' perceived shifts on five feeling scales (i.e. feeling more logical, serious, emotional, fake and different) in pair-wise comparisons between their different languages. A systematic shift was found across the four languages, with participants feeling gradually less logical, less serious, less emotional and increasingly fake when using languages acquired later in life. It can be argued that being able to access a range of languages, also gives one the possibility of the expression of different emotions. As Harris (2006) describes, intense emotions from the formative years will have been encoded in the native language. Nevertheless there are many situations where emotional expression is facilitated by speaking another language. We feel that this occurs frequently when the additional language can circumvent the superego (as embedded in the native language) and so taboo words or emotions can be allowed to be expressed in a way that would not be allowed in the native language. Pavlenko (2005, p. 22) points out that as a Russian-Jewish immigrant to the US, Russian is for her a highly emotional language: "words brim with intimacy and familiarity (...) permeated with memories of my childhood and youth". However, these emotional associations are not systematically positive:

...it is also a language that attempted to constrain me and obliterate me as a Jew, to tie

me down as a woman, to render me voiceless, a mute slave to a hated regime. To

abandon Russian means to embrace freedom. I can talk and write without hearing 
echoes of things I should not be saying. I can be me. English is a language that offered me that freedom (...) (p. 22).

Dewaele (2010) similarly reported that several Arab and Asian participants stated that they switch to English to escape the social taboo in their native languages and cultures. One Chinese participant, Quipinia (Cantonese L1, English L2) reported an incident in which she burst out in English at her parents who know English but with whom she usually speaks Cantonese:

But I remember one time when they were arguing with me and I was soooooooooo angry that I shouted out 'IT'S UNFAIR!!!!' I guess it's regarded quite impolite if I shouted at my parents (you know Chinese Traditional family) but at that point I feel that I had to express my anger and let myself just do it in another language; perhaps I feel I'm another person if I say that in English...(p. 121)

Tehrani \& Vaughan (2009, p. 11) show how bilingual differences and language switching in therapy can increase emotional mastery and how exploring past problems in a new light can be aided by a new language "...where an individual is equally fluent in two languages the most significant factor in increasing the quality and emotional content of the recall is the language and context in which the incident was encoded". Imberti (2007) elaborates further on this theme:

Sometimes the acquisition of a new language can provide a person with the "right expression" for a particular sentiment, and thus can be used as a coping mechanism to express emotionally loaded experiences... a second language served as a vehicle to become more self regulated by finding ways to verbalise feelings that were once censored or restricted by external forces (p. 71)

These examples imply that individuals who are multilingual may have access to a greater emotional range and have a more developed facility for managing plural cultural identities than their monolingual peers. A further implication is that this process should be acknowledged and worked with in the therapeutic encounter.

In the early years, acquisition of the first language can be understood in attachment terms as the main way in which the infant begins to separate from the mother (Winnicott, 1963) as well as the means to relate to others (Stern, 1998). The relationship the child has to 
their acquisition of language and the experience of separation are therefore inextricably linked. This, in part, explains why some people find it so difficult to learn a new language when they migrate. It may excite all types of anxieties around separation and loss - not only from the mother but also from the motherland and mother tongue. Perez Foster (1996) summarises the dual functions of the language operations in the psyche of defense and expression as "the power of bilingualism to both ally itself against the experience of psychic pain and to work in transformative adaptation toward the development of new self experience" (p. 262).

\section{Some findings from earlier research}

One previous small-scale research with bi/multilingual clinicians (Costa, 2010) revealed that bi/multilingual clinicians were using a range of techniques to address their clients' multilingual experience. For example a client could be encouraged to speak his or her own language in specific moments for which "in some cases when clients can't find any similar words in English they may use phrases or words from their language which I may not be able to understand but allows them to express the emotion" (p. 21).

The bi/multilingual clinicians interviewed felt comfortable with this and were able to tolerate not understanding a phrase or sentences initially and then exploring the meaning together, after they had been spoken, in English. The research by Bowker \& Richards (2004) and Stevens \& Holland (2008) with mainly monolingual, English-speaking therapists who work with bi or multilingual clients has provided a variety of examples of ways in which therapists have engaged or struggled with patients where there has been a language differential and some anxiety about the communication. For example, Stevens \& Holland (2008, p. 19) note that when working cross-lingually, counsellors commented that they felt outside of their comfort zone. A therapist interviewed by Bowker \& Richards (2004) commented on her sense of inadequacy and envy as a monolingual clinician working with a client who could speak more than one language, and further comments echoed this: “...it is almost embarrassing, their English is almost more correct than mine is..." (p. 471).

\section{Research question and hypotheses}

In order to build on previous research findings and to shed some light on the complex issue of multilingualism in patient-therapist interactions, the present study will address the following question: "Are there significant differences between monolingual and multilingual therapists in their beliefs, attitudes and practices with multilingual patients?"

\section{Philosophical underpinning}

We have adopted a Critical Realism approach (Bhaskar, 1979) to this research, where understanding of the world through causal explanations or phenomenological meaningmaking are not the only foci for the development of knowledge. Through a Critical Realism approach we have tried to refine our knowledge by using information about the functioning of the brain and observing and describing more fully that information via 
questionnaires and reflective conversations, in order for that full and rich description to provide an evaluative critique of the social phenomena we have observed.

\section{Method}

\section{Participants}

Qualified therapists were contacted by email via one of the author's professional networks inviting them to take part in a piece of research which considered the question: "Are there significant differences between mono-lingual and bilingual/multilingual therapists?" A total of 101 therapists agreed to fill out a short sociobiographical questionnaire. It contained questions about sex, age, nationality, language history and present language use, and theoretical orientation in their therapeutic work. A majority of participants were women $(N=84)$ and 17 male colleagues. The mean age was 46 yrs $(S D$ $=11.8)$, ranging from 25 to 85. The therapists had worked an average of 10.6 years $(S D=$ 9.2), ranging from zero to 40 years in the profession. Participants are generally highly educated: 4 have a Bachelor's degree, 31 a Master's degree, 25 a PG, and 23 a Doctoral degree. This majority of highly educated, mostly female therapists is typical for the profession. For example, The British Association for Counseling and Psychotherapy has a total of 9,671 accredited members of which 8,219 are female and 1,452 are male (May 25th 2012). The participants reported 20 different nationalities, including many participants with double nationalities. The largest group was British $(N=58)$, followed by British and some other nationality $(N=8)$. Other nationalities included American, Chinese, Egyptian, French, German, Greek, Indian, Iranian, Irish, Italian, Polish, Portuguese, Romanian, Russian, South African, Spanish, Swedish, Swiss, Taiwan, Turkish and Venezuelan. Most participants were resident in the UK $(N=93)$. Two thirds of participants $(N=63)$ had lived abroad for longer than 3 months. English was the most frequent L1 $(N=51)$, followed by Turkish $(N=5)$, Greek $(N=4)$, and 21 other L1s. A little under half of the participants had grown up with two L1s from birth $(N=45)$. The sample consists of 19 monolinguals, 30 bilinguals, 22 trilinguals, 20 quadrilinguals, and 11 pentalinguals. Most frequent L2s were English $(N=24)$ and French $(N=18)$. The pattern was similar for the L3 with French $(N=8)$ and Spanish $(N=8)$ as the most frequent languages. The most frequent L4s were Italian $(N=5)$ and Spanish $(N=4)$. No single L5 appeared more than twice. Most therapists used the Humanistic Integrative approach $(N=30)$, followed by the CBT approach $(N=29)$, the Systemic approach $(N$ $=17)$, and the Psychodynamic approach $(N=16)$.

\section{Instrument}

The main questionnaire was exploratory in nature. It contained 27 items in the form of statements with 5-point Likert scales (ranging from "strongly disagree" to "strongly agree") (see appendix A). The items covered linguistic practices with multilingual clients, perceptions and attitudes towards mono- and multilingual interactions, multilingualism and multiculturalism. The questionnaire was first submitted to four experts (two psychologists and two applied linguists) who rated each item and commented on them. After that the questionnaire was pilot-tested with 10 therapists. This led to the deletion of 
some items and the reformulation of others. The final version of the questionnaire was put on-line on Survey monkey and the first author used her contacts in the profession to recruit participants. The questionnaire was anonymous but the last item allowed participants to leave an email address if they agreed to be interviewed on the issues covered in the questionnaire.

\section{Interviews}

Following university ethical approval and the completion of the questionnaires, we conducted a series of interviews with one monolingual, and two multilingual therapists who had given their consent to be contacted in the questionnaires. The aim of the interviews was to provide additional information, which might enrich the data gathered from the questionnaires. Interviews were recorded and transcribed shortly after the recording. All quotes used were checked with interviewees first for their approval to publish. Clearly any qualitative research is potentially influenced by the stances and beliefs of the researcher. This can be regarded as a limitation. It can also be regarded as an inevitable reflection of the complexity of working with individuals' multiple realities. In this research we have chosen to embrace that complexity with an honest attitude to its limitations rather than attempt to eliminate complexity and strive for an elusive simple and neutral position. Our results are an attempt to reflect the full and rich description of our findings.

\section{Results}

\section{Quantitative analysis}

An exploratory factor analysis, using a principal components analysis (PCA) was performed on the 27 items, followed by an independent t-test for post-hoc comparison. Assumptions for factorability of the data were sufficiently met based on Bartlett's test of sphericity, $\left.\chi^{2}=867, d f=351, p<.0001\right)$ supporting the factorability of the correlation matrix, the Kaiser-Meyer-Olkin measure of sampling adequacy, $K M O=.067$, exceeding the recommended value of .6 (Kaiser, 1970), and the anti-image correlation matrix. A varimax solution was used for rotation. The number of factors to retain was determined by examining (a) eigenvalues greater than 1.5; (b) the scree plot of eigenvalues; (c) factor loadings greater than $.30 ;(\mathrm{d})$ interpretability of the factor structures.

The results of the PCA yielded a four-factor solution accounting for $41.2 \%$ of the variance (see Appendix B). Item content suggested that the first factor reflects therapists' attunement towards their bilingual clients (Attunement versus Collusion). This first factor explains $17.2 \%$ of variance. The second factor was about effective communication where language is shared opposed to advantages to working in a second language in therapy. Thus, the factor was named "Shared understanding versus Acting on assumptions" and it explains $8.9 \%$ of variance. The third and fourth factors describe "Freedom of expression versus Difficulty of challenging", and "The distancing effect of the second language versus The advantage of a shared language", explaining $8.1 \%$ and $6.6 \%$ of variance respectively. Factor loadings for the variables are shown in Appendix C. 
Individual factor scores on the various dimensions were used as the dependent variables. An independent t-test showed that the 18 monolingual therapists differed significantly from their 83 bi- or multilingual peers on the first dimension (Attunement versus Collusion) (see table 1). The multilingual therapists are situated more towards the attunement end of the dimension compared to the monolingual therapists (see figure 1).

Table 1

Independent t-test: Monolingual versus Multilingual therapists on the four dimensions

\begin{tabular}{lccc}
\hline Dimension & $t$ & $d f$ & $p$ \\
\hline Attunement versus Collusion & -3.51 & 99 & 0.001 \\
Shared understanding versus Acting on assumptions & -0.42 & 99 & 0.676 \\
Freedom of expression versus Difficulty of challenging & -1.56 & 99 & 0.121 \\
Distancing effect of L2 versus Advantage of a shared language & 0.31 & 99 & 0.76 \\
\hline
\end{tabular}

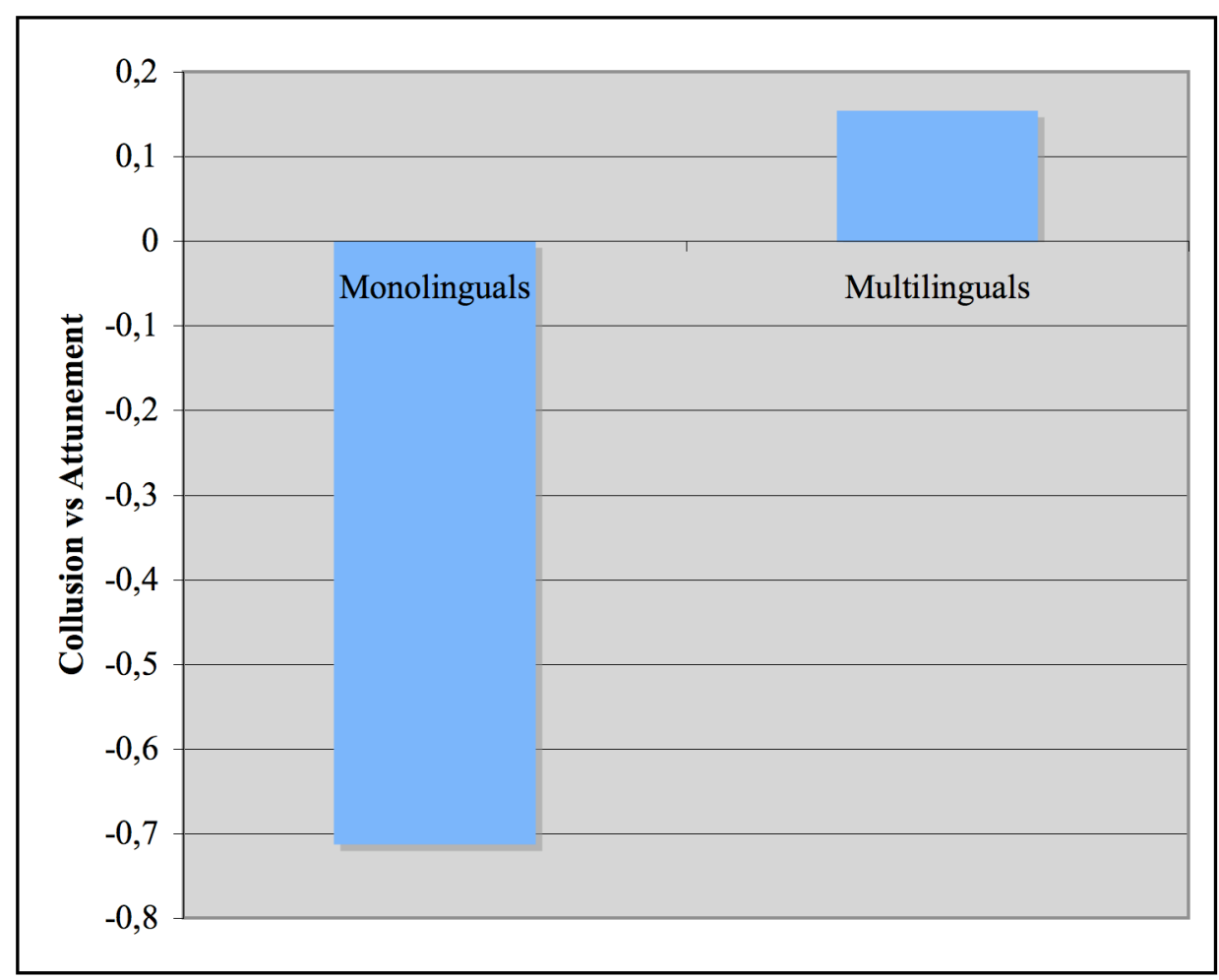

Figure 1. Mean scores on dimension 1: Attunement versus Collusion for both groups of therapists. 


\section{Interviews}

We used the dimension where there had been a significant difference between monolingual and multilingual therapists in the questionnaires to form the basis of the questions in the interviews. The therapists interviewed were from a range of theoretical backgrounds. The monolingual (L1 English) and two multilingual therapists (L2 English) worked within CBT, Systemic and Integrative theoretical frameworks and were all currently employed by the NHS. All of them also had extensive experience of working in the voluntary sector and had worked with monolingual and multilingual clients. Although the conversations were structured around the first factor or dimension (Attunement versus Collusion) where we had found a significant difference between monolingual and multilingual therapists, the therapists sometimes made comments which corresponded to other dimensions which we had hypothesized would be significant and these comments are included in this text.

\section{Dimension 1: Attunement versus Collusion}

As the results from the questionnaire showed, the multilingual therapists tend to view their ability to share a language, or to have a facility for languages with a patient as positive with respect to their capacity for attunement with the client. They are also mindful of the potential for boundary breaches and collusion but do not see this as a negative issue and have adopted strategies to deal with this. The following quotations illustrate their position. Multilingual Therapist 2 (M2):

There is a kind of a familiarity that they (patients) experience with me, that probably they wouldn't with a (native) English speaker or through an interpreter... We know nobody else understands us, it's only us...probably more private, less threatening, less stressful, more relaxed.

Although the following example could be construed as crossing a boundary, this therapist makes it very clear that she is aware of the potential for collusion and is mindful in her practice of how to manage it. M2:

...if somebody's feeling bad in the room, I would very easily say "Let me get you a cup

of... you know some water, can I get you anything?...I'm here to help you and I want

to help you. Would you like a glass of water, you seem in distress?'”...And I don't mind

going and getting it for you. And I think it helps the engagement a lot. 
For the next therapist, it is the act of learning and knowing different languages, which affects her belief that this has made her more attuned to people's levels of understanding. Multilingual Therapist 1 (M1):

How would I find that I communicate differently? I think that if you have to learn various languages for whatever reason, you become much more attuned to what the other person is saying, to try to understand, because...you know what it's like to be a foreigner or in a foreign situation, so you can make that effort and you can be more flexible. (Patients also have) to adapt much more and be more flexible. You are more attuned to whether people understand or don't understand. If I feel they're not understanding. I'll try to get my point across in a different way.

And in answer to the question about what she noticed and what she did, she demonstrated how she applies her beliefs in practice. M1:

I suppose it's sort of a dead-like hue in the eye that they haven't really understood or they haven't caught the thought and followed it in their own mind...I guess I check more... if they've understood or if we've arrived at the same conclusion.

In an unpublished Masters Thesis Bick Nguyen (2012, p. 73) concluded that some of the bilingual therapists she had interviewed were aware of the possibility for overidentification with clients who shared their native language and culture. The multilingual therapists interviewed for this paper are aware of this possibility and the possibility of collusion and show that, in their practice, they are aware and take measures to address it. They also shared a belief that the benefit of reducing a sense of isolation for the patient outweighs the potential for collusion. An example of this is given in the previous section and a following one is included here. M1:

This particular client liked it, that I knew Spain, that I could speak Spanish...and that she wasn't so alone. Being South Asian in England, it's all very difficult, and having wanted to go to Spain, and made this attempt to live in Spain and have that relationship break up, she felt so dislocated. In the therapy she wasn't that alone, she realised there were other people who knew about Spain, knew what was happening. 
The following two extracts show the therapists discussing the issue of disclosure. They take different stances in their practice from a considered and non-collusive position. The differences may be attributed to the different theoretical models they apply in their practice. M1:

...and she (the client) said, "Oh you speak Spanish" and I said "Yes, I do speak

Spanish" So then I had to decide whether I reveal that I'd lived 13 years in Madrid and that I do know Madrid very well or not. I decided not to.

And M2:

They (patients) ask, and I do tell them, I just say that...maybe we don't have the same ethnicity and religion. And it's important because in that context it's actually useful, because they do not perceive me as someone from the authorities or from that more oppressive kind of background. I think it helps me to engage with them. When I feel that they are curious, I might even volunteer, because from my theoretical way we work, we are quite transparent.

This therapist also gives a more detailed example of her practice with relation to patients' behaviour, which invites the therapist into a collusive relationship. M2:

But maybe there is a feeling of appreciation. There is so much appreciation that I'm giving my time to them and it's in Turkish, to get the service in Turkish...And they might also ask things from me, like "Can you do that for me, can you write this letter for me, we are from the same place, can you do this favour for me?" And I...just explain what I can and what I cannot do.

The monolingual therapist interviewed for this paper did not say anything actively about collusion. Perhaps this is due to the fact that therapists, in their professional formation, are careful to avoid being judgemental. The comments by this therapist included in the section on Assumptions could also have fitted easily into this Collusions section. A decision was taken to include her comments in the Assumptions section to mirror the 
therapists' choice of vocabulary. The following quotation is included, however, as it alludes to the possibility of following a lead and imagining a familiarity when that is not the case. Monolingual Therapist (M3):

I'm a little bit suspicious of kind of making assumptions about body language when I don't know what people are saying because I have experience of times when people's body language might have made me think that something was going on when that hasn't actually been the case.

\section{Dimension 2: Shared understanding versus Acting on assumptions}

Even though there was no significant difference between both groups of therapists for this dimension (Shared Understanding versus Assumptions), the interviewed therapists made some interesting comments, and a distinction emerged between the multilingual therapists and the monolingual therapist in terms of beliefs, attitudes, behaviour and practice. The multilingual therapists commented on shared experience and behaviour with clients. M1:

... a monolingual won't have that experience, of going home or thinking that home is elsewhere, or being bored as I was as a child, being dragged back home and thinking "Oh but I really would like to go like everyone else (on holiday) to Portugal."

and shared attitudes. M2:

I feel that because of some of the language they use, or the way they sometimes come in and say, "Hello, how are you?"..."I feel that it's something about that we are in a foreign country and we are...you know where I come from, I know where you come from" kind of an idea, so I think they do relate in a different way.

However, the monolingual therapist felt that the shared language led to shared assumptions. M3:

...when you don't share a language people assume that you probably don't have that shared understanding...People assume you have a shared understanding or shared 
agreement about, you know, what is shameful in culture or whatever, when you share

a language.

This therapist believed that shared assumptions through a shared language was not always useful. Although the word "collusion" was not used, the lack of potential for collusion by the monolingual therapist is highlighted. M3:

I think that sometimes it's actually quite an advantage to not have that assumption and to be able to make explicit a conversation about understandings of culture and ways of thinking or ways of expressing things. It opens up space that actually there may be alternative ways of understanding, that you don't necessarily have to be bound by what you've been brought up with, but you can in some ways choose.

The therapist also added:

I'm very careful not to impose my culture on my client.

For Dimension 3 "Increased freedom of expression versus Challenge less easy" and Dimension 4 "Additional language promotes distance versus Shared language is an advantage" no significant difference emerged in the quantitative analysis between monolingual and multilingual therapists. This lack of difference is echoed in the interviews with the three therapists, although they refer to increased freedom of expression and the benefits of distance when working in an additional language. The fact that the questionnaire revealed no significant difference between monolingual and multilingual therapists in Dimensions 3 and 4 may be explained by the relatively little exposure many therapists have to ideas about emotional expression in multiple languages, as explained in the introduction to this paper. Dimensions 3 and 4 specifically draw on ideas about emotions in multiple languages. However, both the monolingual and multilingual therapists make some observations about these dimensions. Being able to tolerate uncertainty and ambiguity is a key skill for therapists. The gap produced by notknowing can be a source of therapeutic spontaneity and creativity. Winnicott (1971) referred to this as the "potential space". Moreno (1953), the founder of psychodrama, defined spontaneity, the fundamental change agent of psychodrama, as "a new response to an old situation or an adequate response to a new situation" (p. 336). It can be argued that speaking another language is a "new response to an old situation". All the therapists interviewed reflect Nguyen's (2012) observation that a gap across languages can have therapeutic benefits proposing "bilinguals have most likely had opportunities to experience and to live with not-knowing and not understanding. This may be an aid and a 
resource when needing to stay with and to work with ambiguities in the counselling relationship in terms of the language gap" (p. 97).

The following excerpt illustrates how the monolingual therapist describes how she uses the distance produced by the additional language to pay extra attention to body language. M3:

When (using an interpreter) the client is talking in their own language and I don't know what's being said, it gives me space to able to attend to the facial expressions, for example, in a way that might be different from when we're sharing a language.

The following extract shows how the language gap can facilitate ways of challenging the patient as well as allowing for freedom of expression. M2:

I was working with an (English) couple and she'd referred to her mother being a fishwife. I said I don't understand really well, but what is a fishwife? And then she started saying what it is and what she really meant by using that word (...) My intention really was to understand more about what was loaded in that word, which if I was English, for example, I might not have said, "what is a fish wife?" That (not being English) allows me to ask that question.

Her belief is that:

Probably I found out things that maybe a monolingual wouldn't have been able to find out in that way.

Freedom of expression is again referred to with reference to the behaviour of language switching. This differs from the study by Nguyen (2012) who found that therapists believed that the principal function of swapping between two languages was to increase comprehension. The therapists she interviewed did not mention the potential for a change in emotional expression or level of closeness. This is referred to by one of the multilingual therapists below. M2: 
There's a difference in what they're saying by switching, by saying, "you know what I

mean, I'm from this place”. So the act of switching is more important than the switch.

However the monolingual therapist also expresses concern that freedom of expression may be at the expense of safety. M3:

There are times I know when other therapists have done... a testimony on a reliving exercise with somebody and they were speaking in their own language and the therapist didn't understand it. Now, I wouldn't do that, I think there's just too much at the time going on therapeutically that you need to be able to be in touch with for that to be a safe or comfortable process really for the client or the therapist.

\section{Additional Findings}

None of the therapists interviewed had tried inviting their patients to express themselves in their own language (which they did not share) and then have them translate it for them afterwards. In conversation after the interviews, they all felt that (apart from safety considerations) this was an interesting intervention which they would consider. They saw the potential for mending splits and allowing for integration in the therapy room by welcoming the different linguistic identities of the client into the room. It is intriguing that none of the therapists had tried this yet, given the multilinguals' beliefs that the sharing of the patients' language could help the patients to feel less dislocated and isolated. Reference has already been made in this paper to multilingual therapists interviewed by Costa (2010) who endorse inviting other languages into the room. Finally, although there were not specific questions about these topics, two other themes emerged clearly. The first was the fact that the multilingual therapists had trained in English and that this was their professional language. They believed that this affected the way in which they conducted (or didn't) therapy in their native language. The following example illustrates this issue. M1:

Well, when I was thinking about coming to do this interview, I wondered whether I wasn't really a fraud, because although I do speak various languages, I've always been trained in only one, so when I tried to, even when people in Switzerland ask me about the kind of therapy I do, I find it incredibly difficult to explain, because I've never picked up a French textbook about CBT. 
But this therapist also went on to mention another point. M1:

...if you don't have the language and it's not tripping off your tongue and you're having to search for it, you're in the same position as the client.

Perhaps there is an interesting point to be made here about the levelling of power and the increased sense of empathy. This compares with a therapist's response in Costa (2010): "I also think that when people realise English is not my first language either, that changes the balance of power" (p. 19).

The final theme to emerge was about opportunities for learning languages. The multilingual therapists felt that if you are a speaker of a minority language you have little choice but to learn other languages. Whereas for the monolingual therapist even with a will to learn it was not so clear-cut. M3:

Even having worked overseas quite a bit, I find it very difficult to practice the language where I'm English and other people very often want to speak English, so I find it very difficult and to practice and not get it right without people jumping in and speaking to me in English.

\section{Conclusion}

The research set out to discover if there are significant differences between monolingual and multilingual therapists in their beliefs, attitudes and practices with multilingual patients. Although the statistical analysis of the questionnaires showed a significant difference between both groups in only one dimension extracted by principal components analysis (namely Attunement versus Collusion), a variety of points have emerged from the conversations which seem to be applicable to multilingual therapists, others to monolingual therapists only and some which apply equally to both categories.

Multilingual therapists interviewed suggested that they were able to help patients to feel more connected and less isolated although they also mention the importance of attending to boundaries in a way that shapes patients' expectations and the limits of their role. Perez Foster (1996) proposes that when speaking in their shared native language: "both members of the therapeutic couple are pulled into a sensorial space...this experience is similar to the child and early caretaker's sharing of affective states and moods" (p. 71). This clearly heightens the potential for empathy and intimacy but might also lead to possible collusion. This is why the therapists felt that attention to the appropriate setting and maintenance of boundaries and the issue of disclosure is so important. These 
therapists also mentioned the problems of training as therapists in English and the difficulties they experienced working in their native languages in therapy where they did not have access to the professional vocabulary or experience in relating professionally in their native language. Although this was an example of the difficulties the distance of working in an additional language can bring, they also showed some awareness of the potential benefits of working in an additional language. The point has already been made that the limited attention to this issue paid by the therapists reflects the limited input into therapists' training of the psychological and psychotherapeutic functions of multilingualism.

The key point highlighted by the monolingual therapist was the fact that she was free of assumptions and less likely to collude with patients. All the therapists believed that learning a language made them better attuned to other languages and other language learners. They all believed that through working across languages they had learned to think carefully about how they used language, to check understanding and to simplify their language. Although no therapist had tried out inviting other languages in to the therapy they were interested and saw the potential of trying this. They all warned against making any cultural assumptions and in the words of Perez Foster (1996) viewed therapy "as a meaningful co-construction of the patient's life where both members of the dyad are equally involved in the enterprise" (p. 167). Perez Foster (1996, pp. 203-208) describes her ability as a bilingual therapist to work creatively with bilingual clients whose native language she does not know. She uses the term "quasitherapy" to refer to the way in which essential material may be lost working across languages and she illustrates ways in which she has worked with her clients' dual-language worlds with fascinating tips and examples. From our own piece of research the authors would like to make supplementary recommendations for practice.

\section{Recommendations}

We have three recommendations for research, practice, training and supervision. Firstly, it would be useful and interesting for further research to be conducted on language switching in therapy - how it is initiated and what it signifies. The second recommendation relates to practice. This research highlights the need for therapists to pay attention to the way in which the inherent self-disclosure is managed by the therapist who speaks multiple languages. Therapists interviewed for this research have given examples, which are included in this paper, of ways in which they manage this. It is also suggested that therapists consider if, when and how to initiate inviting languages they may not understand into the therapeutic space and the therapeutic implications of such an initiative. Finally, it is suggested that training of psychotherapists needs to include a component on the psychological and therapeutic functions of multi/bilingualism and underlying implications for therapy. Training and supervision for psychotherapists could also include practice for therapists to make formulations in different languages. With increasing numbers of multilingual people now accessing therapeutic services and becoming therapists, it seems timely for the curricula of psychotherapy courses and therapeutic practice for all therapists - mono and multilingual - to be revised in order to take into account the changing profile and language needs of users and providers. 


\section{Acknowledgements}

Our thanks to Professor Rachel Tribe and Dr. Joan Painter for their advice and input on the questionnaire design and ethical issues. Thank you also to Dr. Malcolm Edwards for sharing ideas and experience and to Ruxandra Comanaru for her administration of the questionnaire. Finally, we would like to acknowledge our appreciation of the participants for sharing their thinking with us. 


\section{References}

Altarriba, J., \& Santiago-Rivera, A. L. (1994). Current perspectives on using linguistic and cultural factors in counseling the Hispanic client. Professional Psychology: Research and Practice, 25, 388-397.

Amati-Mehler, J. Argentieri, S., \& Canestri, J. (1993). The babel of the unconscious: Mother tongue and foreign tradition. Madison, CT: International Universities Press.

Bhaskar, R. (1979). The possibility of naturalism: A philosophical critique of the contemporary human sciences. Brighton, UK: Harvester.

Bowker, P., \& Richards, B. (2004). Speaking the same language? Psychodynamic Practice, 10(4), 459-478.

Burck, C. (2004). Living in several languages: implications for therapy. Journal of Family Therapy, 26, 314-339.

Caldwell-Harris, C. L., Tong, J., Lung, W., \& Poo, S. (2011). Physiological reactivity to emotional phrases in Mandarin-English bilinguals. International Journal of Bilingualism 15(3), 329-352.

Costa, B. (2010). Mother tongue or non-native language? Learning from conversations with bilingual/multilingual therapists about working with clients who do not share their native language. Journal of Ethnicity and Inequalities in Health and Social Care, 3(1), 15-24.

De Maesschalck, S. (2012) Linguistic and cultural diversity in the consulting room: A tango between physicians and their ethnic minority patients. Unpublished Doctoral Dissertation, Ghent University.

Dewaele, J.-M. (2010). Emotions in multiple languages. Basingstoke, UK: Palgrave Macmillan.

Dewaele, J.-M., \& Nakano, S. (2012). Multilinguals' perceptions of feeling different when switching languages. Journal of Multilingual and Multicultural Development DOI: $10.1080 / 01434632.2012 .712133$

Harris, C. L. (2006) When is a first language more emotional? In A. Pavlenko (Ed.), Bilingual Minds: Emotional experience, expression, and representation (pp. 257283). Clevedon, UK: Multilingual Matters.

Imberti, P. (2007). Exploring and understanding the language experience of the nonEnglish-speaking immigrant. Families in Society: The Journal of Contemporary Social Services, 88, 67-73.

Kaiser, H. F. (1970). A second generation Little Jiffy. Psychometrika, 35, 401-415.

Li, W. (2000). The bilingualism reader. London, UK: Routledge.

Moreno, J. L. (1953). Who shall survive? Beacon, NY: Beacon House Inc.

Nguyen, B. (2012). Working with monolingual and bilingual clients in the UK when English is not your first language. Unpublished MA Dissertation, Reading University, UK.

Ożańska-Ponikwia, K. (2011). What has personality and emotional intelligence to do with 'feeling different' while using a foreign language? International Journal of Bilingual Education and Bilingualism DOI:10.1080/13670050.2011.616185.

Pavlenko, A. (2005). Emotions and multilingualism. New York, NY: Cambridge University Press. 
Pavlenko, A. (2006). Bilingual selves. In A. Pavlenko (Ed) Bilingual minds: Emotional experience, expression, and representation (pp. 1-33). Clevedon, UK: Multilingual Matters.

Perez Foster, R. (1996). The bilingual self: duet in two voices. Psychoanalytic Dialogues, $3,69-121$.

Perez Foster, R. (1998). The Power of language in the clinical process: Assessing and treating the bilingual person. Northvale, NJ: Aronson.

Santiago-Rivera, A. L., \& Altarriba, J. (2002). The role of language in therapy with the Spanish-English bilingual client. Professional Psychology: Research and Practice, 33, 30-38.

Schrauf, R. W. (2000). Bilingual autobiographical memory: Experimental studies and clinical cases. Culture and Psychology, 6, 387-417.

Stern, D. (1998). The interpersonal world of the infant. London, UK: Karnac.

Stevens, S., \& Holland, P. (2008). Counselling across a language gap: the therapist's experience. Counselling Psychology Review, 23(3), 15-23.

Tehrani, N., \& Vaughan, S. (2009). Lost in translation - using bilingual differences to increase emotional mastery following bullying. Counselling and Psychotherapy Research, 9(1), 11-17.

Winnicott, D. W. (1963). The Maturational process and the facilitating environment. London, UK: Karnac (reprinted 1996).

Winnicott, D. W (1971). Playing and reality. London, England: Routledge.

Wilson, R. J. (2008). 'Another language is another soul': Individual differences in the presentation of self in a foreign language. Unpublished doctoral dissertation. Birkbeck College, University of London. 


\section{Appendix A}

The Likert scale questionnaire. Instruction: "Tell us to what extent you agree with the following statements (Strongly disagree, Disagree, Neutral, Agree, Strongly Agree)"

1. I avoid certain topics when working with people with whom I do not share a first language.

2. Clients with whom I share a first language relate differently from people with whom I do not share a first language.

3. It is easier to form a therapeutic relationship with someone who shares a first language.

4. I use more non-verbal forms of communication with people who do not share my first language.

5. Speakers of more than one language can accommodate more easily in therapeutic work with a client.

6. I consider that the client's language plays a role in how they behave in therapy.

7. I consider that the languages used by the therapists in therapy play a role in how they behave as a therapist.

8. I think there are advantages to using a second language for the client in therapy.

9. I think it is an advantage being familiar with a client's culture.

10. I think being from the same culture as the client is an advantage.

11. I think my ability as a therapist has been improved by working with people who speak a different first language from my own.

12. I think that therapists with bilingual skills are able to understand clients in a different way than therapists who are monolingual.

13. I think therapists who speak more than one language are able to communicate more effectively with clients from different linguistic backgrounds.

14. I think that therapists' ability to speak more than one language attunes them more to cultural differences.

15. I think clients can use a second language as a distancing device in therapy

16. I think therapists can use a second language as a distancing device in therapy

17. I think that therapists who speak more than one language can understand clients whose first language is not that of the therapist.

18. I think the transference is likely to be affected by the client's choice of languages used in therapy.

19. Working with the transference is easier when the therapist and client share a first language.

20. I think my proficiency in my first language affects the way clients view me.

21. I think that the first language of the therapist is not relevant in therapy

22. I think that the first language of the client is not relevant in therapy

23. It is easier to express strong feelings and emotions in a second language

24. I feel that being able to work in a second language would give me more freedom to express myself

25. From my experience, I feel that levels of empathy between clients and therapists are affected by the language in which the therapy takes place

26. I feel less able to challenge clients if I share the same culture or language

27. I feel more able to challenge clients if I share the same culture or language 


\section{Appendix B}

The principal component analysis

\begin{tabular}{|c|c|c|c|}
\hline Component & Eigenvalue & $\%$ of Variance & Cumulative \% \\
\hline & 4.7 & 17.4 & 17. \\
\hline & 2.4 & 8.5 & 2 \\
\hline & & 8.1 & 34.5 \\
\hline & 1. & 6.6 & 41.1 \\
\hline
\end{tabular}

\section{Appendix C}

Varimax rotation of Four-factor solution for the 27 items

\begin{tabular}{|c|c|c|c|c|}
\hline Item & 1 & 2 & 3 & 4 \\
\hline 1. AvoidTopicswitLXusers & $-0 . C$ & 0.4 & 0.4 & -0.0 \\
\hline 2. DifferentRelationLXusers & 0.3 & 0.3 & 0.3 & -0 \\
\hline 3. RelationEasierL1users & $0 . C$ & 0.1 & 0.7 & -0.1 \\
\hline 4. MoreNonVerbalLXusers & $0 . C$ & 0.3 & 0.3 & -0.5 \\
\hline 5. MultilingMoreAccomod & 0.5 & 0.2 & 0.1 & -0.1 \\
\hline 6. LangClientPlaysRole & 0.3 & 0.4 & 0.2 & $0 . \mathrm{C}$ \\
\hline 7. LangTherapPlaysRole & 0.1 & 0.4 & 0.3 & 0.1 \\
\hline 8. AdvantageinUseL2 & 0.2 & 0.4 & -0.3 & $-0 . C$ \\
\hline 9. FamiliarCultisAdvantage & 0 & -0 & 0.5 & $-0 . C$ \\
\hline 10. BelongSameCultAdvant & 0.2 & -0.2 & 0.4 & 0.4 \\
\hline 11. BetterTherapLXusers & 0.4 & 0.3 & $0 . C$ & -0.1 \\
\hline 12. BilingTherapUnderstandDiff & 0.7 & $0 . C$ & 0.1 & $0 . \mathrm{C}$ \\
\hline 13. BilingtherapMoreEffect & 0.8 & $0 . C$ & $0 . C$ & 0.1 \\
\hline 14. BilingTherapMoreAttuned & 0.7 & $0 . C$ & 0.2 & 0.1 \\
\hline 15. ClientsL2DistancingDevice & $0 . C$ & 0.6 & $0 . C$ & $0 . \mathrm{C}$ \\
\hline 16. TherapL2DistancingDevice & $0 . C$ & 0.6 & $-0 . C$ & $0 . C$ \\
\hline 17. BilingTherapUnderstandMore & 0.4 & $0 . C$ & -0.4 & $-0 . C$ \\
\hline 18. TransferenceAffectByLang & 0.2 & 0.4 & $-0 . C$ & 0.6 \\
\hline 19. TransferenceEasierL1users & -0 & 0.2 & $-0 . C$ & 0.6 \\
\hline 20. L1ProfaffectClientview & 0.1 & 0.1 & 0.5 & $0 . \mathrm{C}$ \\
\hline 21. TherapL1 irrelevant & 0.1 & $-0 . C$ & $-0 . C$ & 0.1 \\
\hline 22. ClientL1irrelevant & 0.1 & -0.2 & -0.1 & -0.6 \\
\hline 23. StrongEmoEasierL2 & $0 . C$ & $-0 . C$ & $0 . C$ & 0.2 \\
\hline 24. AbilityworkL2Freedom & 0.2 & $0 . \mathrm{C}$ & 0.2 & $-0 . C$ \\
\hline 25. EmpathyAffectLang & 0.1 & 0.1 & 0.5 & 0.2 \\
\hline 26. LessAbleChallengL1user & -0.3 & 0.2 & $-0 . C$ & $-0 . C$ \\
\hline 27. MoreAbleChallengL1user & -1 & 0.3 & 0.2 & $0 . \mathrm{C}$ \\
\hline
\end{tabular}

Language and Psychoanalysis, 2012 (1), 19-41. 